\title{
summary
}

\section{Symptoms associated with teething}

Macknin ML, Piedmonte M, Jacobs J, Skibinski C. Symptoms associated with infant teething: a prospective study. Pediatrics 2000; 105:747-752

Objective To conduct a large, prospective study of healthy infants to determine which symptoms may be attributed to teething, and attempt to predict tooth emergence from an infant s symptoms.

Design Prospective cohort of 125 well children in a clinic-based paediatric group practice.

Outcome measures Parents recorded daily two tympanic temperatures, presence or absence of 18 symptoms and all tooth eruptions in their infants, from the 4-month well-child visit until the child turned 1 year old.

Results Daily symptom data were available for 19422 child-days and 475 tooth eruptions. Symptoms were only significantly more frequent at 4 days before a tooth emergence, the day of emergence and 3 days after. Increased biting, drooling, gum-rubbing, sucking, irritability, wakefulness, ear-rubbing, facial rash, decreased appetite for solid foods and mild temperature elevation were all statistically associated with teething. Congestion, sleep disturbance, stool looseness, increased stool number, decreased appetite for liquids, cough, rashes other than facial rashes, fever over $102^{\circ} \mathrm{F}$ and vomiting were not significantly associated with tooth emergence. Although many symptoms were associated with teething, no symptom occurred in $>35 \%$ of teething infants, and no symptom occurred $>20 \%$ more often in teething than in non-teething infants. No teething child had a fever of $104^{\circ} \mathrm{F}$ and none had a lifethreatening illness.

Conclusion Many mild symptoms previously thought to be associated with teething were found in this study to be temporally associated with teething. No symptom cluster, however, could reliably predict the imminent emergence of a tooth. Before caregivers attribute to teething any infant's signs or symptoms of a potentially serious illness, other possible causes must be ruled out.

Address for reprints: Michael L Macknin, Department of Pediatrics and Adolescent Medicine, A120, Cleveland Clinic Foundation, Cleveland $\mathrm{OH}$ 44195, USA. E-mail: macknin@ccf.org

\section{Commentary}

A number of childhood upsets and conditions have been attributed to 'teething' over the centuries and a variety of remedies suggested, not all of them innocent of harm.

This paper provides a useful reminder and confirmation of the minor signs and symptoms associated with teething. These include drooling, gum-rubbing, irritability, facial rash, a reduced appetite and mild temperature elevation.

The study has the advantage of being prospective, extending over a period of months and of including a sample of more than 100 healthy infants. Sample size was estimated using power calculations and multivariate analysis was employed. Recordings made by parents related to a total of 19422 child days and related to the eruption of 475 teeth in 89 children. No teeth erupted during the study period in 22 children. Links between teething and symptoms were not always strong and no symptom occurred in more than $35 \%$ of children during the 8-day period around reported tooth emergence.

The study has weaknesses, which are acknowledged by the authors, in that the sample was highly selected. All those included were children registered with a specialist paediatric practice and had at least one parent employed at the Cleveland Clinic Foundation. Many of the children's parents had a medical background and may have been conversant with current understanding of symptoms related to teething. It was also a disadvantage that both eruption and minor signs and symptoms (from a listed series) were reported by the parents. Because of the age of infants studied, the report was confined to eruption of anterior teeth. Eruption of posterior teeth may carry different implications. The study required a high degree of co-operation and assistance from parents. A simpler recording format would be needed if a study was to extend over longer periods or be carried out in more representative samples. A more qualitative approach, using open-ended questions or interviews would also be helpful in determining parents' perceptions of teething and the impact it has on child and family.

Tooth eruption is seen as an important developmental milestone. Paediatricians and paediatric dentists will be familiar with the findings reported here. It is worthwhile, however, to see more objective confirmation of the fact that teething is not a cause of severe systemic upsets and that when these occur alternative causes should be investigated promptly.

RD Holt

Transcultural Oral Health, Eastman Dental Institute, London, UK 